\title{
Automated Acquisition and High-precision Phase Analysis of Vast Numbers of Electron Holograms of Nanoparticles
}

\author{
Yoshio Takahashi ${ }^{1}$, Tetsuya Akashi ${ }^{1}$, Atsuko Sato ${ }^{2}$, Toshiaki Tanigaki ${ }^{1}$, Hiroyuki Shinada ${ }^{1}$ and Yasukazu \\ Murakami $^{2}$ \\ ${ }^{1}$ Hitachi, Ltd., Hatoyama, Saitama, Japan, ${ }^{2}$ Kyushu University, Fukuoka, Fukuoka, Japan
}

Electron holography can reveal the electromagnetic field both within and surrounding materials. Atomic resolution electron holography is a powerful tool for investigating electrostatic potential of nanoparticles including catalyst systems. Since nanoparticles have a small volume, their internal electrostatic potential only generates a weak signal. It is thus essential to improve the signal-to-noise ratio of phase images. One solution for improving the statistical precision of phase analysis is to average numerous images of a nanoparticle. However, if the specimen is sensitive to radiation-induced effects, it is difficult to collect a large number of images of the nanoparticle. Therefore, huge number of holograms are collected from different places of an electron-transparent thin foil with many nanoparticles dispersed on it. If a phase image can be reconstructed from each hologram, identical image projections are classified and averaged to improve signal-to-noise ratio.

We developed an automated acquisition system for collecting a large number of electron holograms [1]. A technique for shifting the electron beam in combination with stage movement allows data to be acquired over a wide area of a TEM-specimen grid. Undesired drift in the hologram position, which may occur during the hologram acquisition, can be corrected in real time by automated detection of the interferencefringe region in a hologram. The shift of interference fringe position in the hologram is not necessary to compensated. Focus can be adjusted to a desired target value in post reconstruction process of the hologram.

Holograms of gold nanoparticles (mean diameter: $5.0 \mathrm{~nm}$ ) dispersed on a carbon foil were collected by the automated acquisition system equipped with a 1.2-MV atomic resolution holography electron microscope [2], which utilize a cold field emission electron gun, a double-biprism electron interferometer, an imaging aberration corrector (CEOS), and a direct CMOS camera (Gatan K2 summit). The system could obtain 1024 holograms, which provided phase maps for more than 500 nanoparticles with a lateral resolution of $0.14 \mathrm{~nm}$, in just one hour. The collected holograms kept the quality for analyzing the phase quantitatively during the acquisition.

The mean inner potentials (MIP) of the collected 140 gold nanoparticles were analyzed. The particle size dependency is plotted in Fig. 1(a), which shows that the phase values are significantly dispersed for the same particle size. The measured MIP dispersion is assumed to consist of both intrinsic dispersion due to non-uniformity of nanoparticles and statistic errors associated with measurement. Averaged values of MIP for width in size of $0.5 \mathrm{~nm}$ are plotted in Fig. 1(b), which was obtained by averaging the MIP values plotted in Fig. 1(a). It appears that MIP decreases only gradually with decreasing particle size down to 4 $\mathrm{nm}$, whereas it anomalously increases in the range below $4 \mathrm{~nm}$. The statistical error and the dispersion due to the non-uniformity were reduced by collecting a large number of holograms.

This size dependency of MIP was investigated by an atomic resolution phase image, which was averaged over 10 images of the same gold nanoparticle as shown in Fig. 2(a). The phase image reveals the polycrystalline nature of the gold nanoparticle as well as the positions of individual atomic columns. An anomalous decrease in phase was observed at a grain boundary in the nanoparticle (Fig. 2(b)). Since the fraction of grain boundaries to particle volume can be increased by reducing particle size, the MIP 
decreases gradually as particle size decreases. On the other hand, as for the atomic column at the nanoparticle border, the distance to the neighboring gold column is reduced with reference to bulk gold crystal. This indicates that the surface of the gold nanoparticle is subjected to a significant compressive stress [3]. The MIP increases with an increase of the density of the gold atom. Since the fraction of number of surface atoms to the total number of atoms in a particle is increased by reducing the particle size, the MIP increases as particle size decreases. This effect will become significant below a particle size of $4 \mathrm{~nm}$.

In conclusion, the automated hologram acquisition system makes it possible to comprehensively analyze electron holograms, including studying nanoparticles with high precision and at atomic-scale resolution [4].
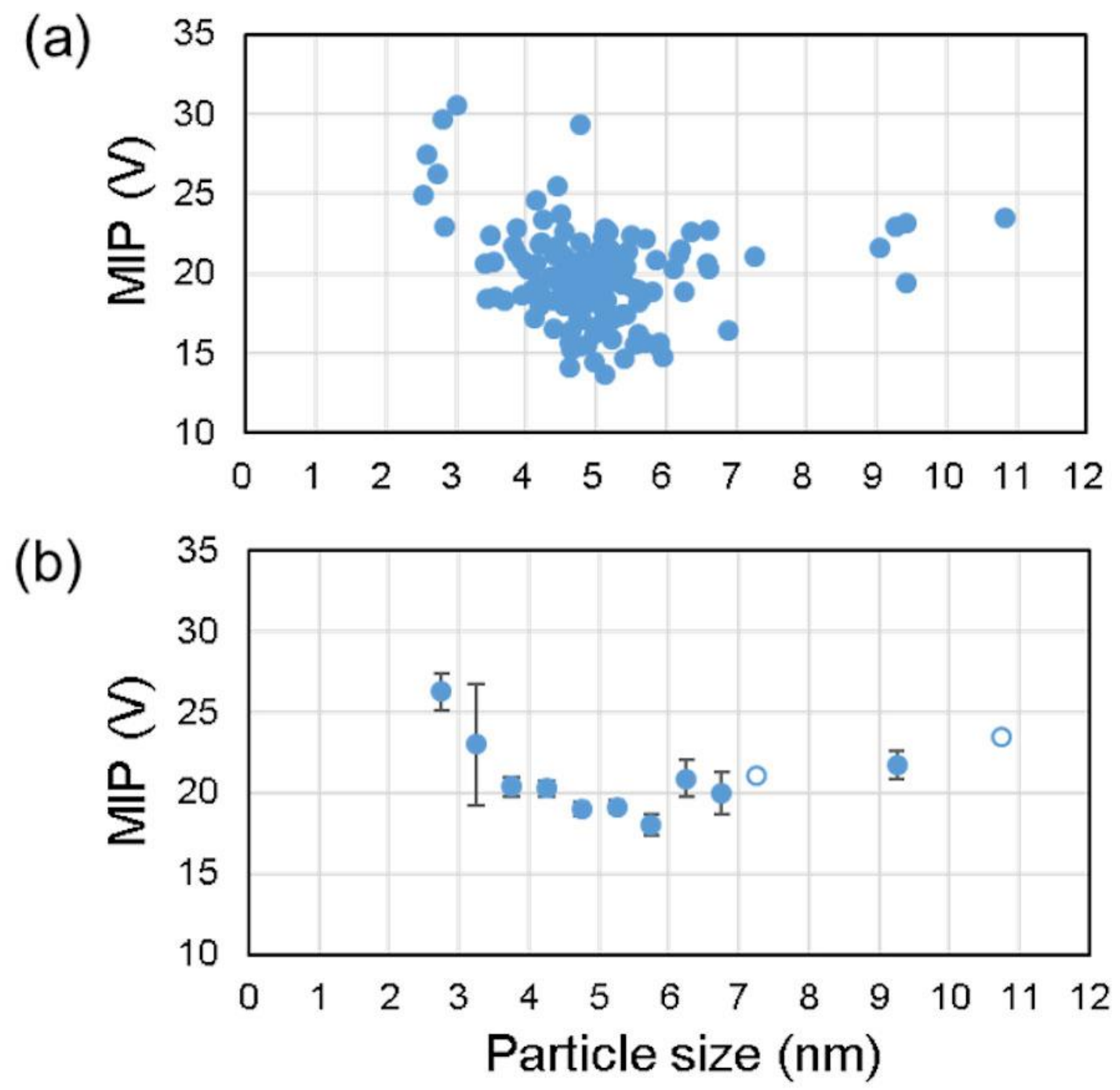

Figure 1. MIP vs. Au particle size. (a) MIP of individual particles is plotted. (b) MIPs are obtained by averaging MIP of individual particles. Range of averaging is a width of $0.5-\mathrm{nm}$. Error bars correspond to standard error. 

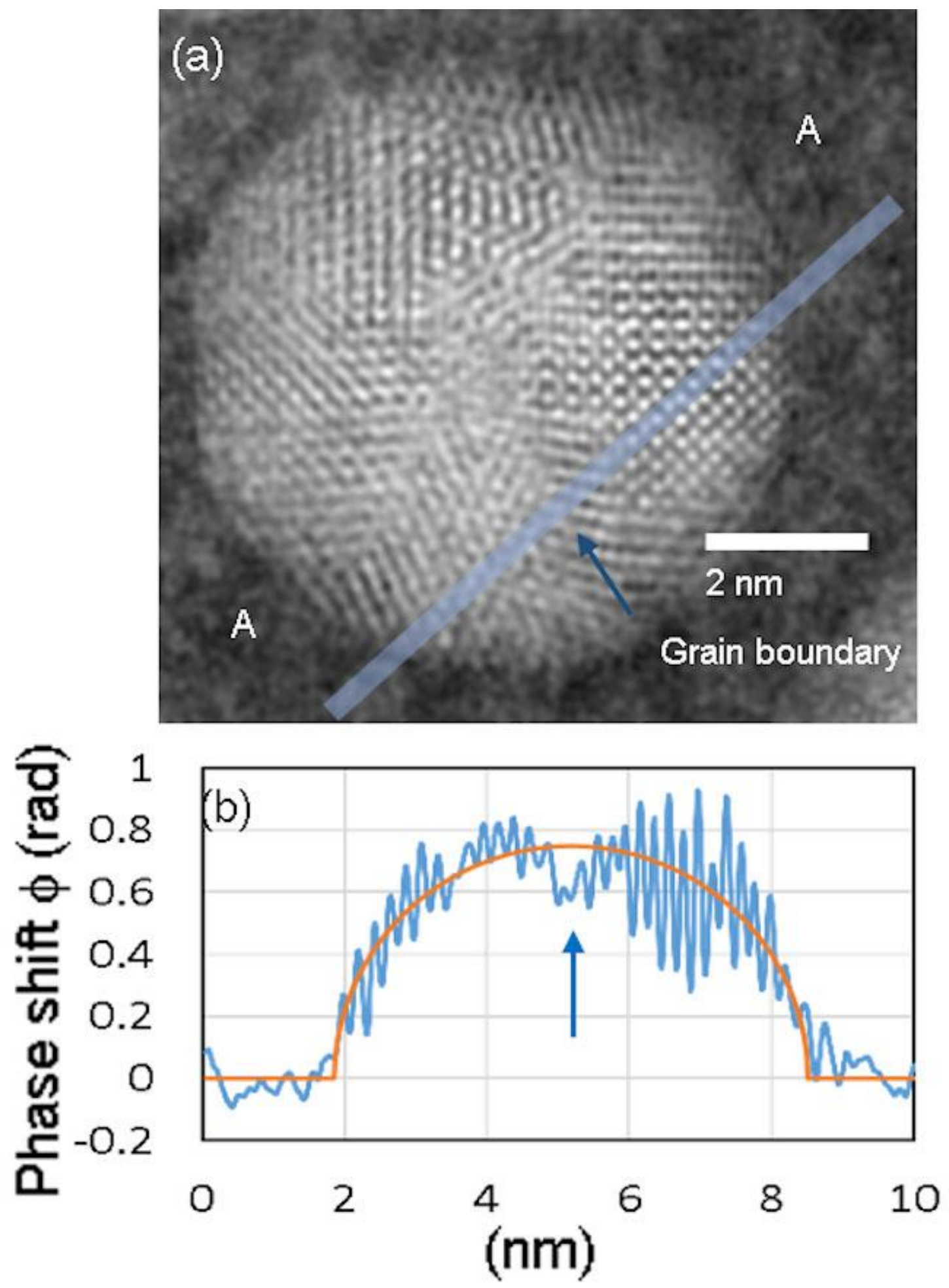

Figure 2. (a) Phase image of Au particle. (b) Line profile along A-A in (a). Arrows indicate a position of grain boundary.

References

[1] Y. Takahashi et al., Microscopy (2020), DOI: 10.1093/jmicro/dfaa004. 
[2] T. Akashi et al., Appl. Phys. Lett., 106 (2015) 074101.

[3] R. Popescu et al., Phys. Rev. B76 (2007) 235411.

[4] This work was supported by JST CREST Grant Number JPMJCR 1664, Japan. 